\title{
New Theoretical Results on Event-by-Event Fluctuations
}

Mark I. Gorenstein*

Bogolyubov Institute for Theoretical Physics, Kiev, Ukraine

E-mail: goren@bitp.kiev.ua

Several theoretical results concerning event-by-event fluctuations are discussed:

(1) a role of the global conservation laws and concept of statistical ensembles;

(2) strongly intensive measures for physical systems with volume fluctuations;

(3) identity method for chemical fluctuations in a case of incomplete particle identification;

(4) the example of particle number fluctuations in a vicinity of the critical point.

9th International Workshop on Critical Point and Onset of Deconfinement - CPOD2014,

17-21 November 2014, ZiF (Center of Interdisciplinary Research), University of Bielefeld, Germany

\footnotetext{
* Speaker.
} 


\section{Introduction}

The study of event-by-event (e-by-e) fluctuations in high-energy nucleus-nucleus $(A+A)$ collisions opens new possibilities to investigate properties of strongly interacting matter (see, e.g., Refs. [1] and [2] and references therein). Specific fluctuations can signal the onset of deconfinement when the collision energy becomes sufficiently high to create the quark-gluon plasma (QGP) at the initial stage of A+A collision [3]. By measuring the fluctuations, one may also observe effects caused by the dynamical instabilities when the expanding system goes through the $1^{\text {st }}$ order transition line between the QGP and the hadron resonance gas [4]. Furthermore, the critical point (CP) of strongly interacting matter may be signaled by characteristic fluctuation pattern [5]. Therefore, e-by-e fluctuations are an important tool for the study of properties of the onset of deconfinement and the search for the $\mathrm{CP}$ of strongly interacting matter.

In this presentation the theoretical progress in several areas related to the study of e-by-e fluctuations is reported. A role of the global conservation laws is discussed in Sec. 2. The strongly intensive measures of e-by-e fluctuations are introduced in Sec. 3. They give a possibility to study e-by-e fluctuations in a physical system when its average size and size fluctuations can not be controlled experimentally. In Sec. 4 a novel procedure, the identity method, is described for analyzing fluctuations of identified hadrons under typical experimental conditions of incomplete particle identification. Finally, in Sec. 5 using the van der Waals equation of state adopted to the grand canonical ensemble formulation we discuss particle number fluctuations in a vicinity of the CP.

\section{Global Conservations Laws}

In this section we illustrate the role of global conservation laws in calculating of e-by-e fluctuations within statistical mechanics. Successful applications of the statistical model to description of mean hadron multiplicities in high energy collisions (see, e.g., Refs. [6] and references therein) has stimulated investigations of properties of the statistical ensembles. Whenever possible, one prefers to use the grand canonical ensemble (GCE) due to its mathematical convenience. The canonical ensemble (CE) should be applied [7] when the number of carriers of conserved charges is small (of the order of 1). The micro-canonical ensemble (MCE) has been used [8] to describe small systems with fixed energy, e.g., mean hadron multiplicities in proton-antiproton annihilation at rest. In all these cases, calculations performed in different statistical ensembles yield different results. This happens because the systems are 'small' and they are 'far away' from the thermodynamic limit (TL). The multiplicities of hadrons produced in relativistic heavy ion collisions are typically much larger than 1. Thus, their mean values obtained within GCE, CE, and MCE are approximately equal each other. One refers here to the thermodynamical equivalence of statistical ensembles in the TL and uses the GCE, as a most convenient one, for calculating the hadron yields. The MCE, CE, and GCE are the most familiar statistical ensembles. In several textbooks (see, e.g., Ref. [9]), the pressure (or isobaric) ensemble has been also discussed. This leads to volume fluctuations around the average value (see Ref. [10]).

The particle multiplicity fluctuations are usually quantified by a ratio of the variance to mean 
value, the scaled variance,

$$
\omega[N] \equiv \frac{\left\langle N^{2}\right\rangle-\langle N\rangle^{2}}{\langle N\rangle}
$$

and are a subject of current experimental activities. In statistical models there is a qualitative difference in properties of a mean multiplicity and a scaled variance of multiplicity distribution. It was recently found $[11,12,13,14]$ that even in the TL corresponding results for the scaled variance are different in different ensembles. Several examples below illustrate this statement.

Let us consider a system which consists of one sort of positively +1 and negatively -1 charged particles (e.g., $\pi^{+}$and $\pi^{-}$mesons) with total charge equal to zero $Q=0$. For the relativistic ideal Boltzmann gas in the volume $V$ at temperature $T$ the GCE results for the average number of $N_{+}$and $N_{-}$and their fluctuations are calculated as:

$$
\left\langle N_{ \pm}\right\rangle_{\text {gce }}=\frac{V}{2 \pi^{2}} \operatorname{Tm}^{2} K_{2}\left(\frac{m}{T}\right) \equiv z, \quad \omega_{\text {gce }}\left[N_{ \pm}\right]=1,
$$

where $m$ is a particle mass, $K_{2}$ is the modified Hankel function, and $z$ is the so called single particle partition function. In the GCE the chemical potential equals zero to satisfy the condition $\langle Q\rangle_{\text {gce }}=0$.

In the CE the charge conservation constrain, $Q=N_{+}-N_{-}=0$, is used for each microscopic state of the system. The CE average number of $\left\langle N_{ \pm}\right\rangle_{\mathrm{ce}}[15]$ and the scaled variance $\omega_{\mathrm{ce}}^{ \pm}[11]$ are calculated as:

$$
\left\langle N_{ \pm}\right\rangle_{\mathrm{ce}}=z \frac{I_{1}(2 z)}{I_{0}(2 z)}, \quad \omega_{\mathrm{ce}}\left[N_{ \pm}\right]=1-z\left[\frac{I_{1}(2 z)}{I_{0}(2 z)}-\frac{I_{2}(2 z)}{I_{1}(2 z)}\right]
$$

In the large volume limit $(V \rightarrow \infty$ corresponds also to $z \rightarrow \infty$ ) one can use an asymptotic expansion of the modified Bessel function, and obtains:

$$
\left\langle N_{ \pm}\right\rangle_{\mathrm{ce}} \cong\left\langle N_{ \pm}\right\rangle_{\text {g.c.e }}=z, \quad \omega_{\mathrm{ce}}\left[N_{ \pm}\right] \cong \frac{1}{2}+\frac{1}{8 z} \cong \frac{1}{2} .
$$

The scaled variance shows a very different behavior than the mean multiplicity: in the large $z$ limit the CE scaled variance is $1 / 2$ of its GCE value, i.e., $\mathrm{CE}$ and GCE are not equivalent for $N_{ \pm}$ fluctuations.

Our second example is the ideal gas of massless neutral Boltzmann particles. We consider the same volume and energy in the MCE and GCE and compare these to formulations for the particle number fluctuations. The approximation $\langle N\rangle_{\text {mce }} \cong\langle N\rangle_{\text {gce }}$ is valid for $\langle N\rangle_{\text {gce }} \gg 1$ and reflects the thermodynamic equivalence of the MCE and GCE. The scaled variances for the multiplicity fluctuations are, however, different in the GCE and MCE [12]:

$$
\omega_{\mathrm{gce}}[N]=1, \quad \omega_{\mathrm{mce}}[N]=\frac{1}{4} .
$$

Thus, despite of thermodynamic equivalence of the MCE and GCE the value of $\omega_{\text {mce }}$ is four times smaller than the scaled variance of the GCE (Poisson) distribution.

We present now the results of the hadron resonance gas (HRG) for the e-by-e fluctuations of negatively charged and positively charged hadrons (see details in Ref. [13]). The corresponding 

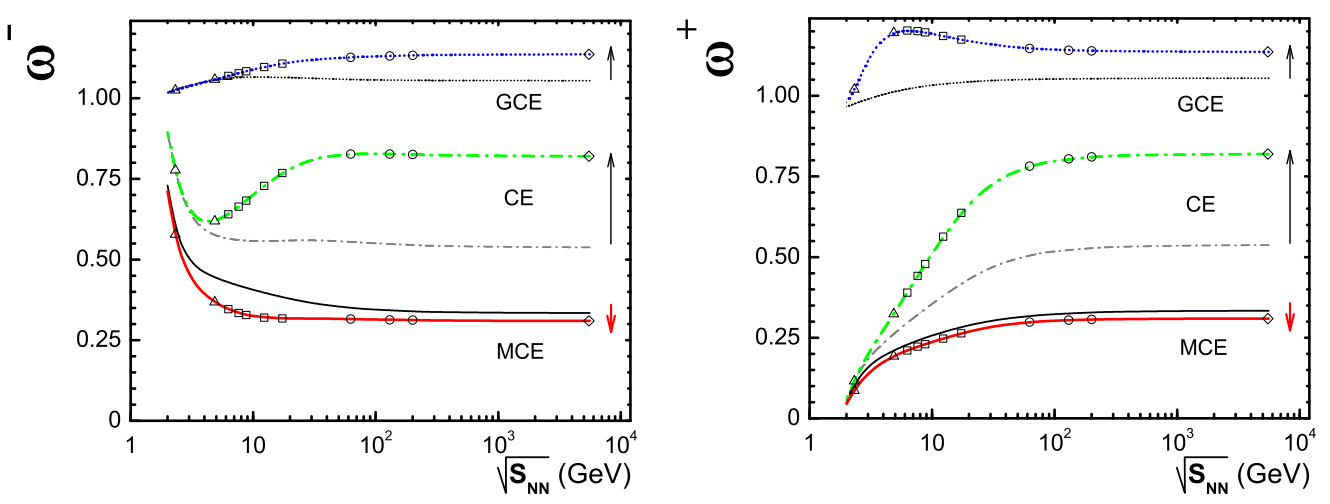

Figure 1: The scaled variances $\omega^{-}$(left) and $\omega^{+}$(right), both primordial and final, along the chemical freeze-out line for central $\mathrm{Pb}+\mathrm{Pb}(\mathrm{Au}+\mathrm{Au})$ collisions. Different lines present the GCE, $\mathrm{CE}$, and MCE results. Symbols at the curves for final particles correspond to the specific collision energies. The arrows show the effect of resonance decays.

scaled variances $\omega^{-}$and $\omega^{+}$are calculated in the GCE, CE, and MCE along the chemical freezeout line in central $\mathrm{Pb}+\mathrm{Pb}(\mathrm{Au}+\mathrm{Au})$ collisions for the whole energy range from SIS to LHC. The model parameters are chosen by fitting the mean hadron multiplicities. Once a suitable set of the chemical freeze-out parameters is determined for each collision energy, the scaled variances $\omega^{-}$ and $\omega^{-}$can be calculated in different statistical ensembles. The results for the GCE, CE, and MCE are presented in Fig. 1 as functions of the center-of-mass energy $\sqrt{s_{N N}}$ of the nucleon pair.

A general concept of the statistical ensembles was suggested in Ref. [16]. The extensive quantities $(V, E, Q) \equiv \vec{A}$ define the MCE. Different statistical ensembles are then constructed using externally given distributions of extensive quantities, $P_{\alpha}(\vec{A})$. The distribution of any observable $O$ in is then obtained in two steps. Firstly, the MCE $O$-distribution, $P_{m c e}(O ; \vec{A})$, is calculated at fixed values of the extensive quantities $\vec{A}$. Secondly, this result is averaged over the external distribution $P_{\alpha}(\vec{A})[16]$ :

$$
P_{\alpha}(O)=\int d \vec{A} P_{\alpha}(\vec{A}) P_{m c e}(O ; \vec{A})
$$

Fluctuations of extensive quantities $\vec{A}$ around their average values depend not on the system's physical properties, but rather on external conditions. The ensemble defined by Eq. (2.6), the $\alpha$-ensemble, includes the standard statistical ensembles as the particular cases. Based on Eq. (2.6) and introducing the scaling volume fluctuations $P_{\alpha}(V)$, an attempt was made in Ref. [17] to extend the statistical model to the hard domain of high transverse momenta and/or high hadron masses.

\section{Strongly Intensive Measures of E-by-E Fluctuations}

A significant increase of transverse momentum and multiplicity fluctuations is expected in the vicinity of the CP. One can probe different regions of the phase diagram by varying the collision energy and the size of colliding nuclei. The possibility to observe signatures of the critical point inspired the energy and system size scan program of the NA61/SHINE Collaboration at the CERN SPS [18] and the low beam energy scan program of the STAR and PHENIX Collaborations at the 
BNL RHIC [19]. In these studies one measures and then compares e-by-e fluctuations in collisions of different nuclei at different collision energies. The average sizes of the created physical systems and their e-by-e fluctuations are expected to be rather different. This strongly affects the observed fluctuations, i.e., the measured quantities would not describe the local physical properties of the system but rather reflect the system size fluctuations (see, e.g., Ref. [20]).

Intensive quantities are defined within the GCE of statistical mechanics. They are independent of the system volume. Strongly intensive quantities introduced in Ref. [21] are, in addition, independent of volume fluctuations. They are the appropriate measures for studies of e-by-e fluctuations in $\mathrm{A}+\mathrm{A}$ collisions and can be defined for two extensive state quantities $A$ and $B$.

There are two families of strongly intensive quantities which depend on the second and first moments of $A$ and $B$ and thus allow to study e-by-e (or state-by-state) fluctuations [21]:

$$
\begin{aligned}
\Delta[A, B] & =\frac{1}{C_{\Delta}}[\langle B\rangle \omega[A]-\langle A\rangle \omega[B]], \\
\Sigma[A, B] & =\frac{1}{C_{\Sigma}}[\langle B\rangle \omega[A]+\langle A\rangle \omega[B]-2(\langle A B\rangle-\langle A\rangle\langle B\rangle)],
\end{aligned}
$$

where

$$
\omega[A] \equiv \frac{\left\langle A^{2}\right\rangle-\langle A\rangle^{2}}{\langle A\rangle}, \quad \omega[B] \equiv \frac{\left\langle B^{2}\right\rangle-\langle B\rangle^{2}}{\langle B\rangle}
$$

and averaging $\langle\ldots\rangle$ is performed over the ensemble of multi-particle states. The normalization factors $C_{\Delta}$ and $C_{\Sigma}$ are required to be proportional to the first moments of any extensive quantities.

There is an important difference between the $\Sigma[A, B]$ and $\Delta[A, B]$ quantities. Namely, in order to calculate $\Delta[A, B]$ one needs to measure only the first two moments: $\langle A\rangle,\langle B\rangle$ and $\left\langle A^{2}\right\rangle,\left\langle B^{2}\right\rangle$. This can be done by independent measurements of the distributions $P_{A}(A)$ and $P_{B}(B)$. The quantity $\Sigma[A, B]$ includes the correlation term, $\langle A B\rangle-\langle A\rangle\langle B\rangle$, and thus requires, in addition, simultaneous measurements of $A$ and $B$ in order to obtain the joint distribution $P_{A B}(A, B)$.

In Ref. [22] a specific choice of the $C_{\Delta}$ and $C_{\Sigma}$ normalization factors was proposed. It makes the quantities $\Delta[A, B]$ and $\Sigma[A, B]$ dimensionless and leads to $\Delta[A, B]=\Sigma[A, B]=1$ in the independent particle model (IPM).

Note that the $\Phi$ measure, introduced some time ago [23], belongs to the $\Sigma$ family within the current classification scheme. An extension of the strongly intensive fluctuation measures for higher order cumulants were suggested in recent paper [24].

The calculations of $\Delta$ and $\Sigma$ measures were performed in Ref. [25]. First experimental results on $\Delta\left[P_{T}, N\right]$ and $\Sigma\left[P_{T}, N\right]$ in $\mathrm{p}+\mathrm{p}$ and $\mathrm{Pb}+\mathrm{Pb}$ collisions have been reported in Refs. [26, 27]. The analysis of $\pi^{+}$and $\pi^{-}$fluctuations and correlations from resonance decays in terms of $\Sigma\left[\pi^{+}, \pi^{-}\right]$ is done in Ref. [28]. Note that an idea to use the e-by-e fluctuations of particle number ratios to estimate the number of hadronic resonances was suggested for the first time in Ref. [29].

\section{Incomplete Particle Identification}

The effect of particle misidentification distorts the measured fluctuation quantities. The identity variable was introduced in Ref. [30], and in Ref. [31] a new experimental technique called the 
identity method was proposed. In Refs. [32,33] this method was extended and all the second moments as well as the higher moments of the joint multiplicity distribution of particles of different types are calculated in spite of the effects of incomplete identification. The results $[32,33]$ can be used for an arbitrary number $k \geq 2$ of hadron species. It is assumed that particle identification is achieved by measuring the particle mass $m$. Since any measurement is of finite resolution, we deal with continuous distributions of observed masses denoted as $\rho_{j}(m)$, with $j=1, \ldots, k \geq 2$, and normalized as $\int d m \rho_{j}(m)=\left\langle N_{j}\right\rangle$.

The identity variables $w_{j}(m)$ are defined as

$$
w_{j}(m) \equiv \frac{\rho_{j}(m)}{\rho(m)}, \quad \rho(m) \equiv \sum_{i=1}^{k} \rho_{i}(m) .
$$

We introduce the quantities

$$
W_{j} \equiv \sum_{i=1}^{N(n)} w_{j}\left(m_{i}\right), \quad W_{j}^{2} \equiv\left(\sum_{i=1}^{N(n)} w_{j}\left(m_{i}\right)\right)^{2}, \quad W_{p} W_{q} \equiv\left(\sum_{i=1}^{N(n)} w_{p}\left(m_{i}\right)\right) \times\left(\sum_{i=1}^{N(n)} w_{q}\left(m_{i}\right)\right)
$$

with $1 \leq p<q \leq k$, and define their event averages as

$$
\left\langle W_{j}^{2}\right\rangle=\frac{1}{N_{\mathrm{ev}}} \sum_{n=1}^{N_{\mathrm{ev}}} W_{j}^{2}, \quad\left\langle W_{p} W_{q}\right\rangle=\frac{1}{N_{\mathrm{ev}}} \sum_{n=1}^{N_{\mathrm{ev}}} W_{p} W_{q},
$$

where $N_{\mathrm{ev}}$ is the number of events, and $N(n)=N_{1}(n)+\cdots+N_{k}(n)$ is the total multiplicity in the $n$th event. The quantities $\left\langle W_{j}^{2}\right\rangle$ and $\left\langle W_{q} W_{p}\right\rangle$ can be calculated as follows [32]

$$
\begin{aligned}
& \left\langle W_{j}^{2}\right\rangle=\sum_{i=1}^{k}\left\langle N_{i}\right\rangle\left[u_{j i}^{2}-\left(u_{j i}\right)^{2}\right]+\sum_{i=1}^{k}\left\langle N_{i}^{2}\right\rangle\left(u_{j i}\right)^{2}+2 \sum_{1 \leq i<l \leq k}\left\langle N_{i} N_{l}\right\rangle u_{j i} u_{j l}, \\
& \left\langle W_{p} W_{q}\right\rangle=\sum_{i=1}^{k}\left\langle N_{i}\right\rangle\left[u_{p q i}-u_{p i} u_{q i}\right]+\sum_{i=1}^{k}\left\langle N_{i}^{2}\right\rangle u_{p i} u_{k i}+\sum_{1 \leq i<l \leq k}\left\langle N_{i} N_{l}\right\rangle\left[u_{p i} u_{q l}+u_{p l} u_{q i}\right] .
\end{aligned}
$$

where $(s=1,2)$

$$
u_{j i}^{s} \equiv \frac{1}{\left\langle N_{i}\right\rangle} \int d m w_{j}^{s}(m) \rho_{i}(m), \quad u_{p q i} \equiv \frac{1}{\left\langle N_{i}\right\rangle} \int d m w_{p}(m) w_{q}(m) \rho_{i}(m) .
$$

Equations (4.4) and (4.5) can be transformed a system of $k+k(k-1) / 2$ linear equations for the $k$ second moments $\left\langle N_{j}^{2}\right\rangle$ with $j=1, \ldots, k$ and $k(k-1) / 2$ correlators $\left\langle N_{p} N_{q}\right\rangle$ with $1 \leq p<q \leq k$.

The above procedure eliminates the effect of misidentification and provides the values of all the second moments $\left\langle N_{j}^{2}\right\rangle$ and $\left\langle N_{p} N_{q}\right\rangle$ in a model-independent way, as they would be obtained in an experiment in which each particle is uniquely identified. This method was generalized to determine third and higher moments of the multiplicity distributions in events consisting of an arbitrary number of different particle species in Ref. [33]. Measurements of the third and higher moments of e-by-e fluctuations are expected to be more sensitive for the search of the $\mathrm{CP}$ in A+A collisions $[34,35]$. First results on fluctuations based on the identity method were presented in Refs. [27, 36, 37]. 


\section{Fluctuations at the Critical Point}

In this section we consider the van der Waals (VDW) equation of state with both repulsive $(b>0)$ and attractive $(a>0)$ terms (see, e.g., Refs. [38, 39]):

$$
p(V, T, N)=\frac{N T}{V-b N}-a \frac{N^{2}}{V^{2}}=\frac{n T}{1-b n}-a n^{2} .
$$

It gives an example of a system with the CP. The equation of state (5.1) was suggested in 1873, and for his work van der Waals obtained the Nobel Prize in physics in 1910. The particle proper volume parameter in Eq. (5.1) equals to $b=4 \cdot\left(4 \pi r^{3} / 3\right)$ with $r$ being the corresponding hard sphere radius of particle.

The VDW equation of state contains a $1^{\text {st }}$ order liquid-gas phase transition and CP. The thermodynamical quantities at the $\mathrm{CP}$ are equal to [39]: $T_{c}=8 a /(27 b), n_{c}=1 /(3 b), p_{c}=a /\left(27 b^{2}\right)$. The VDW equation (5.1) can be then rewritten in the following dimensionless (reduced) form: $\widetilde{p}=8 \widetilde{T} \widetilde{n} /(3-\widetilde{n})-3 \widetilde{n}^{2}$, where $\widetilde{n}=n / n_{c}, \widetilde{p}=p / p_{c}$, and $\widetilde{T}=T / T_{c}$. In the dimensionless presentation the VDW equation has a universal form independent of the values of $a$ and $b$, and the CP is transformed to $\widetilde{T}_{c}=\widetilde{p}_{c}=\widetilde{n}_{c}=1$. To describe the phase coexistence region below the critical temperature the VDW isotherms should be corrected by the well known Maxwell construction of equal areas.

The VDW equation (5.1) can be transformed to the GCE [40]. Note that chemical potential $\mu$ regulates $\langle N\rangle_{\text {gce }}$, i.e., $N$ plays the role of conserved charge, and no antiparticles are introduced. The scaled variance for the particle number fluctuations is then [40]:

$$
\omega[N] \equiv \frac{\operatorname{Var}[N]}{\langle N\rangle}=\frac{T}{n}\left(\frac{\partial n}{\partial \mu}\right)_{T}=\left[\frac{1}{(1-b n)^{2}}-\frac{2 a n}{T}\right]^{-1}=\frac{1}{9}\left[\frac{1}{(3-\widetilde{n})^{2}}-\frac{\widetilde{n}}{4 \widetilde{T}}\right]^{-1} .
$$

It is clearly seen from Eq. (5.2) that in the VDW gas the repulsive interactions suppress the particle number fluctuations, whereas the attractive interactions lead to their enhancement. Note that for $a=0$ the scaled variance (5.2) is reduced to the result for the excluded volume model obtained earlier in Ref. [41].

In Fig. 2 left the lines of constant values of $\omega[N]$ are shown on the $(\widetilde{n}, \widetilde{T})$ phase diagram outside of the mixed phase region.

At any fixed value of $\widetilde{T}$, the particle number fluctuations approach to those of the ideal gas, i.e., $\omega[N] \cong 1$, at $\widetilde{n} \rightarrow 0$, and become small, $\omega[N] \ll 1$, at $\widetilde{n} \rightarrow 3$. As it should be, the scaled variance is always positive for all possible values of $\widetilde{n}$ and $\widetilde{T}$ outside of the mixed phase region.

At the $\mathrm{CP}(\widetilde{T}=\widetilde{n}=1)$ the scaled variance of the particle number fluctuations diverges in the GCE. Introducing the quantities $\tau=\widetilde{T}-1 \ll 1$ and $\rho=\widetilde{n}-1 \ll 1$ and keeping only the lowest orders of them one finds

$$
\omega[N] \cong \frac{4}{9}\left[\tau+\frac{3}{4} \rho^{2}+\tau \rho\right]^{-1}
$$

Note that parameter $\tau$ is positive, while $\rho$ can be both positive and negative.

The VDW equation of state permits the existence of metastable phases of super-heated liquid and super-cooled gas. The lines of constant values of $\omega[N]$ are shown on the $(\widetilde{n}, \widetilde{T})$ phase diagram 

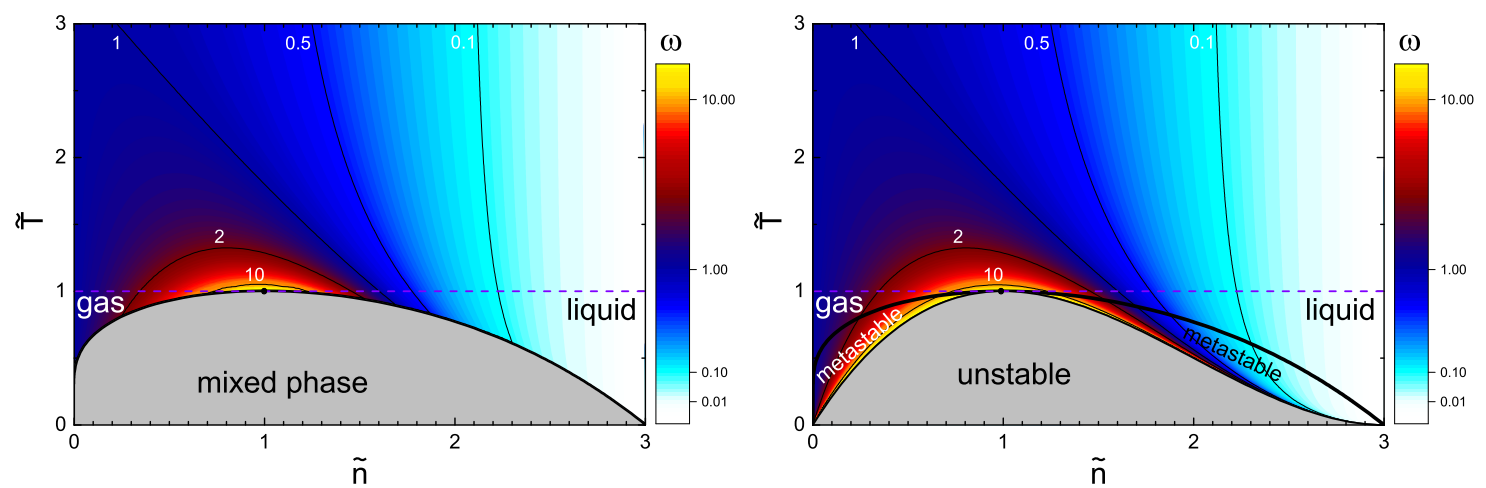

Figure 2: Left: The lines of constant values of the scaled variance $\omega[N]$ are shown on the $(\widetilde{n}, \widetilde{T})$ phase diagram, outside of the mixed phase region. Right: The lines of constant values of the scaled variance $\omega[N]$ are shown on the $(\widetilde{n}, \widetilde{T})$ phase diagram for both stable and metastable pure phases. The boundary between stable and metastable phases is depicted by the thick black line, and the unstable region is depicted by the gray area.

in Fig. 2 right for both stable and metastable pure phases, while the unstable region is depicted by the gray area. The scaled variance diverges at the boundary between the metastable and unstable regions, where $\partial \widetilde{p} / \partial \widetilde{n}=0$,

Details of the calculations inside the mixed phase are presented in Ref. [40]. In a vicinity of the critical point inside the mixed phase we introduce $0<t=1-\widetilde{T} \ll 1$ and find $\omega[N] \cong 16 t^{-1} / 9$. Thus, the scaled variance $\omega[N]$ diverges at the critical point reached from both outside and inside the mixed phase.

\section{Summary}

1. The global conservation of energy and conserved charges influences the measured e-by-e fluctuations of all observables. Thus, to calculate these fluctuations within statistical mechanics one should properly choose the statistical ensemble. The GCE, CE, and MCE are only some particular examples. Real situation may correspond to very different externally given distributions of the volume $V$, energy $E$, and conserved charge(s) $Q$.

2. To avoid the trivial contributions from the system size fluctuations the strongly intensive measures of e-by-e fluctuations should be used. These $\Delta[A, B]$ and $\Sigma[A, B]$ measures are constructed from the second moments $\left\langle A^{2}\right\rangle,\left\langle B^{2}\right\rangle$, and $\langle A B\rangle$ of two extensive quantities $A$ and $B$.

3. A special procedure, the identity method, should be used to calculate the chemical fluctuations in a case of the incomplete particle identification. It solves the misidentification problem for the second and higher moments. Thus the joint multiplicity distribution of particles of different types (a number of species $k \geq 2$ ) can be uniquely reconstructed.

4. The van der Waals equation of state is considered in the GCE, and particle number fluctuations are calculated. The scaled variance $\omega[N]$ diverges at the the critical point. Admitting a presence of metastable states, one observes that the scaled variance $\omega[N]$ diverges also at the boundary between the metastable and unstable regions, where $\partial p / \partial n=0$. 
Acknowledgements. This work was supported by the Humboldt Foundation and by the Program of Fundamental Research of the Department of Physics and Astronomy of National Academy of Sciences, Ukraine

\section{References}

[1] V. Koch, in Relativistic Heavy Ion Physics, Landold-Börnstein Volume I/23, edited by R. Stock (Springer, Berlin, 2010).

[2] M. Gazdzicki, M. I. Gorenstein, and P. Seyboth, Int. Journ. Mod. Phys. E 23, 1430008 (2014).

[3] M. Gazdzicki, M. I. Gorenstein and S. Mrowczynski, Phys. Lett. B 585, 115 (2004); M. I. Gorenstein, M. Gazdzicki and O. S. Zozulya, Phys. Lett. B 585, 237 (2004).

[4] I. N. Mishustin, Phys. Rev. Lett. 82, 4779 (1999); Nucl. Phys. A 681, 56c (2001); H. Heiselberg and A. D. Jackson, Phys. Rev. C 63, 064904 (2001).

[5] M. Stephanov, K. Rajagopal, and E. Shuryak, Phys. Rev. Lett. 81, 4816 (1998); Phys. Rev. D 60 , 114028 (1999); M. Stephanov, Acta Phys. Polon. B 35, 2939 (2004); V. Koch, A. Majumder and J. Randrup, Phys. Rev. Lett. 95, 182301 (2005); V. Koch, A. Majumder, and J. Randrup, Phys. Rev. C 72, 064903 (2005).

[6] J. Cleymans, H. Oeschler, K. Redlich, and S. Wheaton, Phys. Rev. C 73, 034905 (2006); F. Becattini, J. Manninen, and M. Gaździcki, ibid. 73, 044905 (2006); A. Andronic, P. Braun-Munzinger, and J. Stachel, Nucl. Phys. A 772, 167 (2006).

[7] F. Becattini, Z. Phys. C 69, 485 (1996); F. Becattini and U. Heinz, Z. Phys. C 76, 269 (1997); J. Cleymans, K. Redlich, and E. Suhonen, Z. Phys. C 51, 137 (1991). M.I. Gorenstein, M. Gaździcki, and W. Greiner, Phys. Lett. B 483, 60 (2000); M.I. Gorenstein, A.P. Kostyuk, H. Stöcker, and W. Greiner, Phys. Lett. B 509, 277 (2001).

[8] F. Becattini and L. Ferroni, Eur. Phys. J. C 35, 243 (2004); 38, 225 (2004); V.V. Begun, L. Ferroni, M.I. Gorenstein, M. Gaździcki, and F. Becattini, J. Phys. G 32, 1003 (2006); F. Becattini and L. Ferroni, Eur. Phys. J. C 51, 899 (2007); 52, 597 (2007).

[9] Yu.B. Rumer and M.Sh. Ryvkin, Thermodynamics, Statistical Physics, and Kinetics, Nauka, 1972 (in Russian); K.B. Tolpygo, Thermodynamics and Statistical Physics, Kiev University, 1966 (in Russian).

[10] M. I. Gorenstein, J. Phys. G 35, 125102 (2008).

[11] V.V. Begun, M. Gaździcki, M.I. Gorenstein, and O.S. Zozulya, Phys. Rev. C 70, 034901 (2004).

[12] V.V. Begun, M.I. Gorenstein, A.P. Kostyuk, and O.S. Zozulya, Phys. Rev. C 71, 054904 (2005).

[13] V.V. Begun, M. Gaździcki, M.I. Gorenstein, M. Hauer, B. Lungwitz, and V.P. Konchakovski, Phys. Rev. C 76, 024902 (2007).

[14] V.V. Begun, M.I. Gorenstein, and O.S. Zozulya, Phys. Rev. C 72, 014902 (2005); A. Keränen, F. Becattini, V.V. Begun, M.I. Gorenstein, and O.S. Zozulya, J. Phys. G 31, S1095 (2005); F. Becattini, A. Keränen, L. Ferroni, and T. Gabbriellini, Phys. Rev. C 72, 064904 (2005); J. Cleymans, K. Redlich, and L. Turko, Phys. Rev. C 71, 047902 (2005); J. Phys. G 31, 1421 (2005); V.V. Begun and M.I. Gorenstein, Phys. Rev. C 73, 054904 (2006); V.V. Begun, M.I. Gorenstein, A.P. Kostyuk, and O.S. Zozulya, J. Phys. G 32, 935 (2006); V.V. Begun, M.I. Gorenstein, M. Hauer, V.P. Konchakovski, and O.S. Zozulya, Phys. Rev. C 74, 044903 (2006); M. Hauer, V.V. Begun, and M.I. Gorenstein, Eur. Phys. J. C 58, 83 (2008); M. Hauer, Phys. Rev. C 77, 034909 (2008). 
[15] J. Rafelski and M. Danos, Phys. Lett. B 97, 279 (1980).

[16] M.I Gorenstein and M. Hauer, Phys. Rev. C 78 041902(R) (2008).

[17] V.V. Begun, M. Gaździcki, and M.I. Gorenstein, Phys. Rev. C 78, 024904 (2008).

[18] M. Gazdzicki [NA61/SHINE Collaboration], J. Phys. G 36, 064039 (2009).

[19] G. Odyniec [STAR Collaboration], J. Phys. G 35, 104164 (2008).

[20] V. P. Konchakovski, M. I. Gorenstein, E. L. Bratkovskaya, and W. Greiner, J. Phys. G 37, 073101 (2010).

[21] M. I. Gorenstein and M. Gazdzicki, Phys. Rev. C 84, 014904 (2011).

[22] M. Gazdzicki, M. I. Gorenstein, and M. Mackowiak-Pawlowska, Phys. Rev. C 88, 024907 (2013).

[23] M. Gaździcki and St. Mrowczyński, Z. Phys. C 54, 127 (1992).

[24] E. Sangaline, arXiv:1505.0026 [nucl-th].

[25] K. Grebieszkow, Acta Phys. Polon. B 43, 1333 (2012); V. V. Begun, V. P. Konchakovski, M. I. Gorenstein and E. Bratkovskaya, J. Phys. G 40, 045109 (2013); M. I. Gorenstein and K. Grebieszkow, Phys. Rev. C 89, 034903 (2014); M. I. Gorenstein and M. Rybczynski, Phys. Lett. B 730, 70 (2014).

[26] T. Anticic et al. [NA49 Collaboration], Phys. Rev. C 79, 044904 (2009); T. Czopowicz [NA61/SHINE Collaboration], arXiv:1503/0161; PoS (CPOD-2014).

[27] P. Seyboth [NA49 and NA61/SHINE Collaborations], Proceedings Contribution arXiv:1402.4619; see also slides at https://atlaswww.hep.anl.gov/ismd13/;

[28] V. V. Begun, M. I. Gorenstein, and K. Grebieszkow, arXiv:1409.3023[nucl-th], J. Phys. G, in print.

[29] S. Jeon and V. Koch, Phys. Rev. Lett. 83, 5435 (1999).

[30] M. Gazdzicki, Eur. Phys. J. C 8, 131 (1999).

[31] M. Gazdzicki, K. Grebieszkow, M. Maćkowiak, and S. Mrówczyński, Phys. Rev. C 83, 054907 (2011).

[32] M. I. Gorenstein, Phys. Rev. C 84, 024902 (2011).

[33] A. Rustamov and M.I. Gorenstein, Phys. Rev. C 86, 044906 (2012).

[34] A. Stephanov, Phys. Rev. Lett. 102, 032301 (2009); 107, 052301 (2011).

[35] C. Athanasiou, K. Rajagopal, and M. Stephanov, Phys. Rev. D 82, 074008 (2010).

[36] T. Anticic et al. [NA49 Collaboration], Phys. Rev. C 89, 054902 (2014).

[37] M. Mackowiak-Pawlowska [NA49 and NA61/SHINE Collaborations], PoS (CPOD 2013), 048; M. Mackowiak-Pawlowska and A. Wilczek, arXiv:1402.0707 [hep-ph].

[38] W. Greiner, L. Neise, and H. Stöcker Thermodynamics and Statistical Mechanics, 1995 Springer-Verlag New York, Inc.

[39] L. D. Landau and E. M. Lifshitz, Statistical Physics (Oxford: Pergamon) 1975.

[40] V. Vovchenko, D. V. Anchishkin, and M. I. Gorenstein, arXiv:1501.0378[nucl-th].

[41] M. I. Gorenstein, M. Hauer, and D. O. Nikolajenko, Phys. Rev. C 76, 024901 (2007). 